\title{
Estimation of Image Corruption Inverse Function and Image Restoration using a PSO-based Algorithm
}

\author{
Mohammd Pourmahmood \\ Aghababa \\ Electrical Engineering Department, \\ Islamic Azad University Mamaghan \\ Branch, Mamaghan, Iran
}

\author{
Amin Mohammadpour \\ Shotorbani \\ Power Engineering Department, \\ Azerbaijan University of Tarbiat \\ Moallem, Tabriz, Iran
}

\author{
Easa Narimani \\ Faculty of Management \\ Islamic Azad University Ahar Branch, \\ Ahar, Iran
}

\begin{abstract}
A new method is proposed to estimate corruption function inverse of a blurred image. This technique can be used for restoring similar corrupted images. For linear position invariant procedure, the corruption process is modeled in the spatial domain by convolving the image with a point spread function (PSF) and addition of some noises into the image. It is assumed that a given artificial image is corrupted by a degradation function, represented by the PSF, and an additive noise. Then a filter mask (as a candidate for the corruption function inverse) is calculated to restore the original image from the corrupted one, with some accuracy. Calculating a suitable filter mask is formulated as an optimization problem: find optimal coefficients of the filter mask such that the difference between the original image and filter mask restored image to be minimized. Particle swarm optimization (PSO) is used to compute the optimal coefficients of the filter mask. Square filter masks are considered. A comparison between different exciting methods and the proposed technique is done using simulations. The simulation results show that the proposed method is effective and efficient. Since the proposed method is a simple linear technique, it can be easily implemented in hardware or software.
\end{abstract}

\section{Keywords}

Corruption Function, Filter Mask, Image Restoration, Particle Swarm Optimization.

\section{INTRODUCTION}

Fundamental issue in image enhancement or restoration is blur removal in the presence of observation noise. Recorded images almost always represent a degraded version of the original scene. There are many sources, such as the turbulence and aerosol sources in the atmosphere or spatio-temporal movements and intensity scintillations, which cause to image degradation. A systematic approach for restoration of blurred images models the blurred image as a convolution between the original image and the point spread function (PSF). In such a case, simple deconvolution techniques (such as a Wiener filtering) can be used to restore the image [1]. However, the main difficulty is the need for a reliable knowledge of the PSF. The reason for this difficulty is that in most practical remote sensing situations this data is not known in priori [2].
The process of restoring an image by using a degradation function, which has been estimated in some way sometimes, is called blind deconvolution (due to the fact that the true degradation function is seldom known completely).

There has been extensive works on blind deconvolution over the past 20 years, including survey articles [3, 4]. Existing blind deconvolution methods can be categorized into two main classes: the methods which separate PSF identification as a disjoint process from restoration $[5,6,7]$, and the methods which combine PSF identification and restoration in one procedure $[8,9,10,11]$.

Methods in the first class tend to be computationally simpler. For example, Chalmond [6] has proposed isolate specific features in the image, such as sharp edge elements, and then estimated the PSF from them, assuming radial symmetry. However, a main drawback of this method is the assumption that the shapes of all the extracted edges can be modeled as ideal step functions in the original image. No criterion is employed to assess the best stepedge from the set of sharp edge elements in the degraded image. Methods in the second class use iterative methods to approximate the blur extent $[3,4,8-11]$. They often formulate parametric models for both the image and the blur. The parameters are estimated in each step and used in the next iteration. A Gaussian function is used often to approximate the PSF [8-11]. A shortcoming of these methods is the requirement for a good initial guess of the PSF. The resulting accuracy of the estimated PSF and the quality of the restored images depend on the accuracy of the initial guess. The convergence speed of algorithm depends on this guess. Also, in order to obtain a reasonable restored image these methods require one to impose suitable constraints on the PSF and the image.

The mentioned methods have some difficulties and restrictions. They can be just applied for some especial cases and resulting in low accuracy of the obtained PSF and the restored image. Also, when some unknown noises are added to the degraded image, the performance of mentioned methods is decreased. In order to solve the mentioned problems, we propose an optimal filter mask that can produce restored image from degraded image and represent the inverse of the degradation function with the additive noise. For reaching this aim, first an artificial image is corrupted with some degraded functions and additive noises. Then an optimal filter mask is calculated using particle swarm optimization (PSO) algorithm. 
PSO is a population-based searching technique which has been proposed in 1995 by Kennedy and Eberhart [18]. Its development is based on the observations of social behavior of animals such as bird flocking, fish schooling and swarm theory. Compared with genetic algorithm (GA), PSO has some attractive characteristics. First of all, PSO has memory (the knowledge of good solutions is retained by all particles) whereas in GA previous knowledge of the problem is destroyed once the population is changed. Secondly, PSO has constructive cooperation between particles, i.e. particles in the swarm share their information. In PSO, each particle makes use of the best position encountered by itself and that of its neighbors to position itself toward and optimal solution. The performance of each particle is evaluated using a predefined objective function, which encapsulates the characteristics of the optimization problem [16].

In this paper, PSO algorithm is used to find the elements of a filter mask. The computed filter mask minimizes the difference between artificially degraded image and obtained restored image by the filter mask. In other words, the optimal filter mask is a suitable inverse of the image corruption caused by PSF and additive noises, which can be used to restore other similar corrupted images. This method is completely new and its effectiveness is shown by simulations. The simulations are performed using Matlab software. The existing blurring functions, such as motion, average and unsharp, are used as image degradation functions. Salt \& pepper, localvar and gaussian noises are also used to construct artificially corrupted images. The inverse of corruption function is modeled as a simple linear square filter mask. The optimal values of the filter mask elements are computed using PSO technique. The obtained results are compared to the results of some well-known existing filters, such as geometric mean filter, harmonic mean filter and alpha-trimmed mean filter, to demonstrate the efficiency and applicability of the proposed technique.

This paper is organized as follows. In Section II, the problem of image restoration is briefly discussed. In Section III, the procedure of PSO method is explained. Section IV explains the proposed method for estimation of the corruption function inverse. In section $\mathrm{V}$, some simulations are used to illustrate the efficiency of the proposed method. Finally, this paper ends with some conclusions and future works in Section VI.

\section{PROBLEM FORMULATION}

In this section, we formulate the problem of image restoration. As Figure 1 shows, the image corruption process is modeled as a degradation function, which is represented by its PSF and an additive noise $n(x, y)$, operated on an input image $f(x, y)$ to produce a corrupted image $\mathrm{g}(\mathrm{x}, \mathrm{y})$. The standard image restoration problem is as follows: given $\mathrm{g}(\mathrm{x}, \mathrm{y})$, the objective of restoration is to obtain an estimate $\hat{f}(x, y)$ of the original image such that the estimated image to be as close as possible to the original input image. In general, the more knowledge about the PSF and $\mathrm{n}(\mathrm{x}, \mathrm{y})$ will give closer estimation to $\mathrm{f}(\mathrm{x}, \mathrm{y})$.

For a linear position-invariant process the corruption function is given in the spatial domain by

$$
\mathrm{g}(\mathrm{x}, \mathrm{y})=\operatorname{PSF}(\mathrm{x}, \mathrm{y}) * \mathrm{f}(\mathrm{x}, \mathrm{y})+\mathrm{n}(\mathrm{x}, \mathrm{y})
$$

where $\operatorname{PSF}(\mathrm{x}, \mathrm{y})$ is the spatial representation of the degradation function and the symbol "*" indicates the convolution operator.

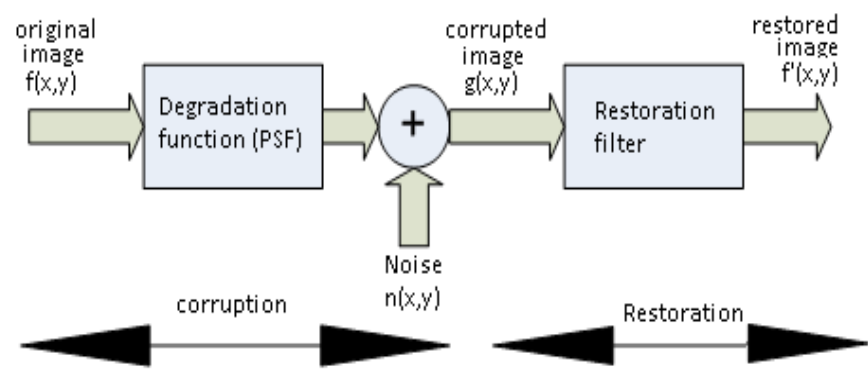

Figure 1. Model of the image corruption/restoration process.

In many applications, there is no complete knowledge about the PSF and the noise. If we can first estimate a suitable inverse of the corruption function (the influence of both PSF and noise), then we can use the obtained corruption model inverse to restore other degraded images that have been corrupted in similar ways. It can be performed as follows. Feed an artificial (typical) image to the degradation function and add it some noises to obtain the artificial corrupted image. Find a good filter mask such that it can be represented as a suitable inverse of the corruption function. For linear spatial filtering, the above process consists simply of moving the filter mask window from point to point in the corrupted image $\mathrm{g}(\mathrm{x}, \mathrm{y})$.In general, linear filtering of an image (here the corrupted image) of size $\mathrm{M} \times \mathrm{N}$ with a filter mask of size $\mathrm{m} \times \mathrm{n}$ is given by

$\hat{f}(x, y)=\sum_{s=-a}^{a} \sum_{t=-b}^{b} w(s, t) f(x+s, y+t)$

where $\mathrm{a}=(\mathrm{m}-1) / 2$ and $\mathrm{b}=(\mathrm{n}-1) / 2$.

Finding $m \times n$ coefficients of the filter mask by PSO is the objective of this paper. Therefore, in the following section a brief explanation of PSO mechanism is given.

\section{PARTICLE SWARM OPTIMIZATION}

A particle swarm optimizer is a population based stochastic optimization algorithm modeled after the simulation of the social behavior of bird flocks. PSO is similar to genetic algorithm (GA) in the sense that both approaches are population-based and each individual has a fitness function. Furthermore, the adjustments of the individuals in PSO are relatively similar to the arithmetic crossover operator used in GA. However, PSO is influenced by the simulation of social behavior rather than the survival of the fittest. Another major difference is that, in PSO each individual benefits from its history whereas no such mechanism exists in GA. In a PSO system, a swarm of individuals (called particles) fly through the search space. Each particle represents a candidate solution to the optimization problem. The position of a particle is 
influenced by the best position visited by itself (i.e. its own experience) and the position of the best particle in its neighborhood. When the neighborhood of a particle is the entire swarm, the best position in the neighborhood is referred to as the global best particle and the resulting algorithm is referred to as a gbest PSO. When smaller neighborhoods are used, the algorithm is generally referred to as a lbest PSO. The performance of each particle (i.e. how much close the particle is to the global optimum) is measured using a fitness function that varies depending on the optimization problem.

The global optimizing model proposed by Shi and Eberhart [16] is as follows:

$$
\begin{aligned}
& \mathrm{v}_{\mathrm{i}+1}=\mathrm{w} \times \mathrm{v}_{\mathrm{i}}+\text { RAND } \times \mathrm{c}_{1} \times\left(\mathrm{P}_{\text {best }}-\mathrm{x}_{\mathrm{i}}\right)+\operatorname{rand} \times \mathrm{c}_{2} \times \\
& \left(\mathrm{G}_{\text {best }}-\mathrm{x}_{\mathrm{i}}\right) \\
& \mathrm{x}_{\mathrm{i}+1}=\mathrm{x}_{\mathrm{i}}+\mathrm{v}_{\mathrm{i}+1}
\end{aligned}
$$

where $v_{i}$ is the velocity of particle $i, x_{i}$ is the particle position, $w$ is the inertial weight. $c_{1}$ and $c_{2}$ are the positive constant parameters, Rand and rand are the random functions in the range [0,1], Pbest is the best position of the $\mathrm{i}^{\text {th }}$ particle and Gbest is the best position among all particles in the swarm.

The inertia weight term, w, serves as a memory of previous velocities. The inertia weight controls the impact of the previous velocity: a large inertia weight favors exploration, while a small inertia weight favors exploitation $[16,18]$. As such, global search starts with a large weight and then decreases with time to favor local search over global search [16].

It is noted that the second term in equation (1) represents cognition, or the private thinking of the particle when comparing its current position to its own best. The third term in equation (1), on the other hand, represents the social collaboration among the particles, which compares a particle's current position to that of the best particle [17]. Also, to control the change of particles' velocities, upper and lower bounds for velocity change is limited to a user-specified value of Vmax. Once the new position of a particle is calculated using equation (2), the particle, then, flies towards it [16]. As such, the main parameters used in the PSO technique are: the population size (number of birds); number of generation cycles; the maximum change of a particle velocity Vmax and w.

Generally, the basic PSO procedure works as follows: the process is initialized with a group of random particles (solutions). The $i^{\text {th }}$ particle is represented by its position as a point in search space. Throughout the process, each particle moves about the cost surface with a velocity. Then the particles update their velocities and positions based on the best solutions. This process continues until stop condition(s) is satisfied (e.g. a sufficiently good solution has been found or the maximum number of iterations has been reached).

\section{ESTIMATION OF THE CORRUPTION FUNCTION INVERSE BY PSO ALGORITHM}

The problem of finding the corruption function inverse is explained in section 2. It is mentioned that for this goal, first an artificial image is passed from the degradation function (in practice this degradation function is not mathematically known and it is produced by motion turbulences, camera distortions, environmental conditions and so on) then an additive noise is also added to get an artificial corrupted image. Using these two images and by means of PSO an optimal filter mask is found. PSO is used to find $\mathrm{m} \times \mathrm{n}$ coefficients of the filter mask, iteratively. For implementing a PSO, a cost function must be defined as a criterion for minimization. Absolute difference between the original image and the image estimated by filter mask is maybe the natural cost function. Therefore, it is defined by

cost function $=(\hat{f}(x, y)-f(x, y))^{2}$

The proposed procedure for finding the optimal mask is given as a flowchart in Figure 2.

As shown in Figure2, after defining the cost function, the population of particles is initialized. Each particle represents coefficients of a candidate filter mask. The size of the filter determines the size of the particle. In this paper, the filters are assumed to be square sized (i.e. the size of the mask is $n \times n$ ), so the particle size is equal to $\mathrm{n} 2$. Other parameters such as initial speed vector, population size, inertial coefficient, acceleration constants and the value of the maximum number of the iterations are also initialized. Afterwards, each particle is used to calculate the corresponding corrupted image $\hat{\mathrm{f}}(\mathrm{x}, \mathrm{y})$, using Eq. (2). The corrupted image is then used to compute the cost function for each particle, using Eq. (5). The best particle and the local best particles and their corresponding costs are saved. Then, the new positions, as the new better solutions (filter masks), are produced by Eqs. (3) and (4). Finally the stop condition is checked; if it is satisfied the algorithm ends otherwise the process is iterated. This process continues until a termination condition is selected (in this paper a maximum value for iterations as the stopping criterion). When the algorithm is stopped, the best particle is the best solution and is the optimal filter mask which can approximate the corruption function inverse. Now, using the computed filter mask, one can be restore other similar corrupted image. The proposed method is linear, simple and intelligent that its performance is evaluated by some simulations in the subsequent section.

\section{SIMULATION RESULTS}

In this section, the efficiency of the proposed method is evaluated using different simulations. For this purpose, we use Matlab software Image Processing Toolbox to produce degradation functions and additive noises. Motion, average and unsharp blurring functions are selected as degradation functions. To construct artificially corrupted images, salt \& pepper, localvar and gaussian noises are used as the additive noises. The obtained 


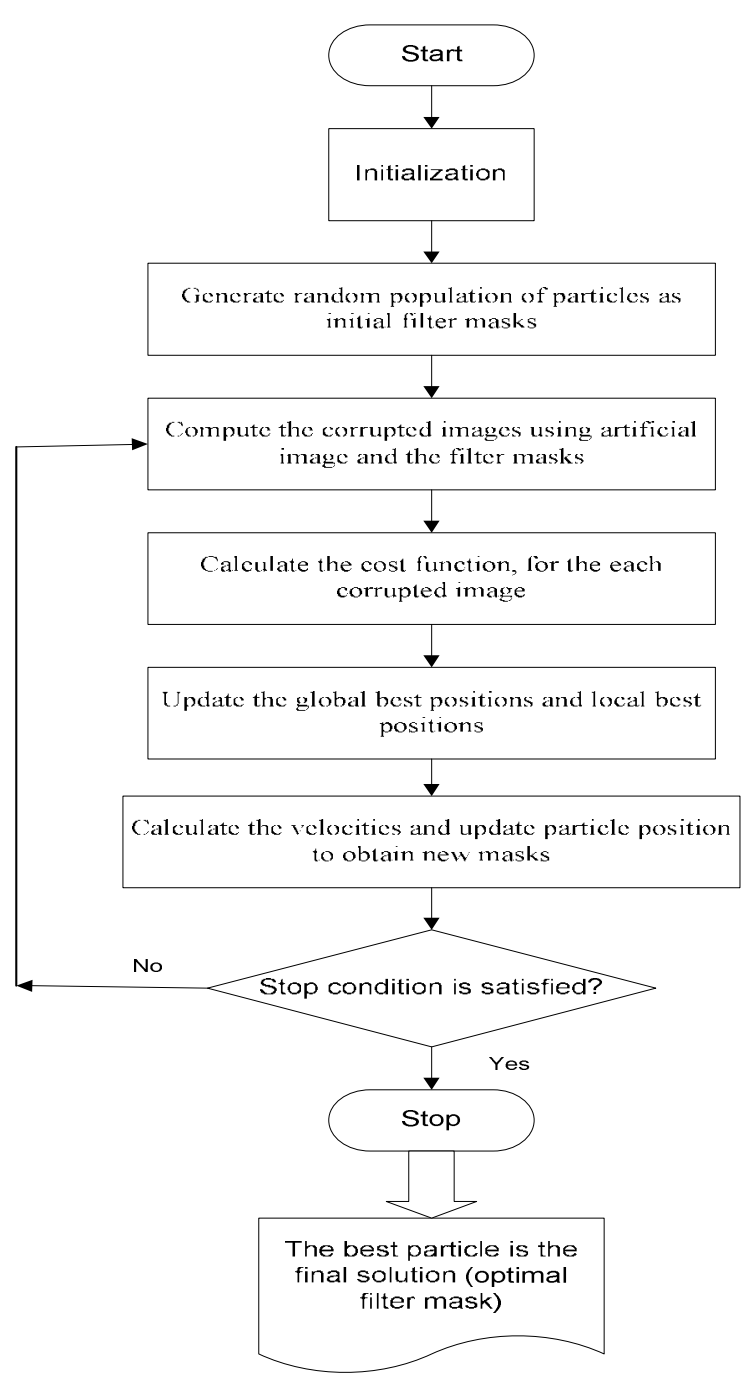

Figure 2. The flowchart of PSO procedure for finding the optimal filter mask

results are compared to the results of some well-known existing filters, such as geometric mean filter, harmonic mean filter and alpha-trimmed mean filter, to demonstrate the efficiency and applicability of the proposed technique. In all simulations population size of the swarm is considered to be 10 . The maximum number of iterations is selected equal to 100 . Inertial coefficient, w, is set as a gradually decreasing function of iterations. Both parameters $\mathrm{c} 1$ and $\mathrm{c} 2$ are chosen equal to 2 . Since the PSO algorithm has a random nature, it is run 100 times for each case study and the best result of them is selected. The mask size is considered to be $n 2=25$. ISNR criterion [19] is used to show the quality of the restored image.

$I S N R=10 \log \left(\frac{\sum \sum(g(x, y)-f(x, y))^{2}}{\sum \sum(f(x, y)-\hat{f}(x, y))^{2}}\right)$

One can see that the larger ISNR, the better performance is achieved.
In the first case, the lena image is corrupted by motion function and localvar noise. Figure 3 shows the simulation results. ISNR criterion results are appeared in Table 1 . One can see that the results of the proposed method are better than the results of the other traditional filters.
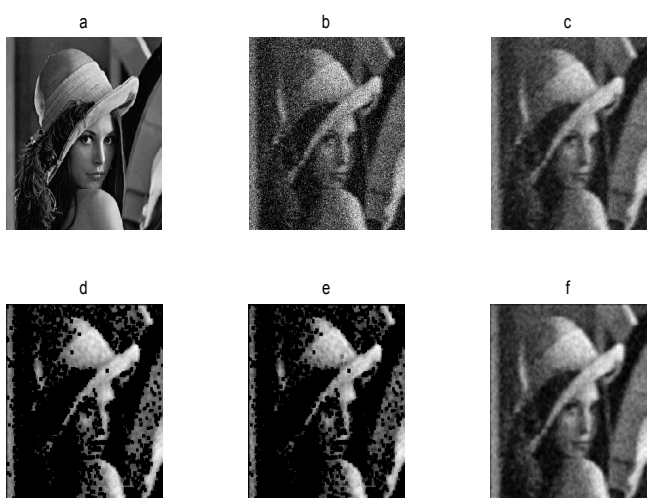

Figure 3. Results of different filters and PSO based technique for restoration of images corrupted by motion function and localvar noise, a) original image; b) degraded image; c) restored image by

PSO; d) restored image by geometric mean filter; e) restored image by harmonic mean filter; f) restored image by alphatrimmed mean filter

TABLE 1. ISNR Criterion for Restoration of Images Corrupted by Motion Function and Localvar Noise

\begin{tabular}{|c|c|}
\hline Method & ISNR \\
\hline PSO found filter mask & 9.85 \\
\hline geometric mean filter & -21.97 \\
\hline harmonic mean filter & -23.05 \\
\hline alpha-trimmed mean filter & -8.07 \\
\hline
\end{tabular}

As Second case, the lena image is corrupted by average function and salt \& pepper noise. Simulation results are depicted in Figure 4. Also, ISNR criterion results are shown in Table 2. It is obvious that the PSO found linear filter mask works better than linear and nonlinear filters.

As final experimentation, the efficiency of the proposed method is validated by corrupting lena image with unsharp function and gaussian noise. The results of simulations are illustrated in Figure 5. Also, Table 3 shows ISNR criterion results. The results reveal the good performance of the PSO found optimal linear filter mask compared to the other conventional filters.

\section{CONCLUSIONS}

In this paper, a new intelligent method for solving image restoration problem is introduced. Using artificially corrupted images, a linear filter mask is found to act as the inverse of the image corruption process. For finding the filter mask coefficients, an optimization problem is formulated and solved by the PSO technique. Once the optimal filter mask is found, it can be used to 
restore other similar corrupted images, without having any knowledge about degradation function or additive noises. The performance of the proposed method is evaluated using several simulations. Since the proposed method is a simple linear procedure, it can be implemented in hardware or software easily. The future work can be focused on using other intelligent tools, such as artificial neural networks and fuzzy logic, for image restoration problem. We can use an artificial neural network to approximate the relationship between a corrupted image and original image. Then, the neural network will be used to restore the other similar corrupted images. Also, we can use other PSO optimized filters, not only the mask filters, in order to restore degraded noisy images.
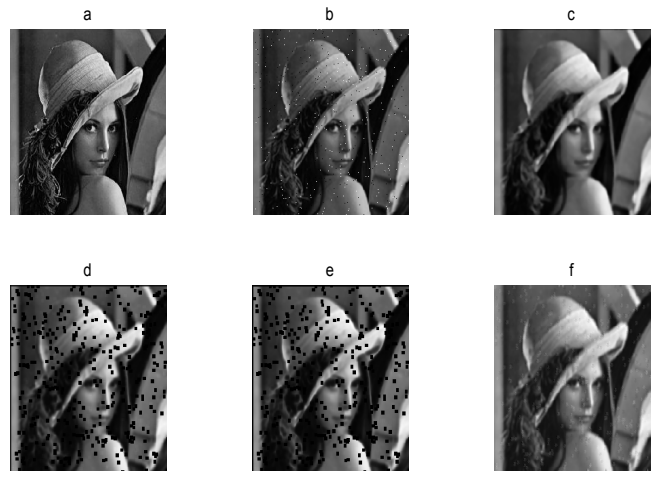

Figure 4. Results of different filters and PSO based technique for restoration of images corrupted by average function and salt $\&$ pepper noise, a) original image; b) degraded image; c) restored image by PSO; d) restored image by geometric mean filter; e) restored image by harmonic mean filter; $f$ ) restored image by alpha-trimmed mean filter
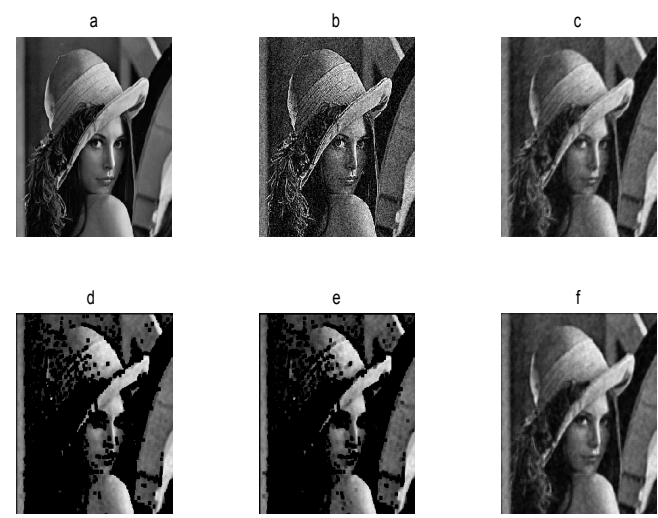

Figure 5. Results of different filters and PSO based technique for restoration of images corrupted by unsharp function and gaussian noise, a) original image; b) degraded image; c) restored image by PSO; d) restored image by geometric mean filter; e) restored image by harmonic mean filter; f) restored image by alpha-trimmed mean filter
TABLE 2. ISNR Criterion for Restoration of Images Corrupted by Avarage Function and Salt \& Pepper Noise

\begin{tabular}{|c|c|}
\hline Method & ISNR \\
\hline PSO found filter mask & 12.13 \\
\hline geometric mean filter & -26.90 \\
\hline harmonic mean filter & -28.16 \\
\hline alpha-trimmed mean filter & -20.83 \\
\hline
\end{tabular}

TABLE 3. ISNR Criterion for Restoration of Images Corrupted by Unsharp Function and Gaussian Noise

\begin{tabular}{|c|c|}
\hline Method & ISNR \\
\hline PSO found filter mask & 13.08 \\
\hline geometric mean filter & -15.89 \\
\hline harmonic mean filter & -17.04 \\
\hline alpha-trimmed mean filter & -1.61 \\
\hline
\end{tabular}

\section{REFERENCES}

[1] Kopeika, N. S. 1998. A System Engineering Approach to Imaging, SPIE Optical Engineering Press, 2nd ed. Bellingham, Washington.

[2] O. Shacham, O. Haik, and Y. Yitzhaky, "Blind restoration of atmospherically degraded images by automatic best stepedge detection", Pattern Recognition Letters 28, 2007, pp. 2094-2103

[3] Jalobeanu, A., Zerubia, J., and Blanc-Feraud, L. 2007. Blind Image Deconvolution: Theory and Applications, Taylor \& Francis/CRC Press. 2007.

[4] D. Kundur, and D.Hatzinakos. "Blind image deconvolution", IEEE Signal Process. Mag. vol. 13, 1996, pp. 43-64.

[5] A. S. Carasso, "Direct blind deconvolution", SIAM J. Appl. Math., vol. 61 (6), 2001, pp. 1980-2007.

[6] B. Chalmond, "PSF estimation for image deblurring", Graphical Models Image Process, vol. 53 (4), 1991, pp. 364372.

[7] Y. Yitzhaky, R. Milberg, S. Yohaev, and N.S. Kopeika, "Comparison of direct blind deconvolution methods for motion-blurred images", Appl. Opt. vol. 38 (20), 1999, pp. 4325-4332.

[8] Nishi, K., and Ando, S. 1994. Blind superresolving image recovery from blur invariant edges. In Proceedings of the International Conference on Acoustics, Speech, Signal Processing, Vol. 5, Adelaide, Australia, pp. 85-88.

[9] G. Pavlovic, and A. M. Tekalp, "Maximum likelihood parametric blur identification based on a continuous spatial domain model", IEEE Trans. Image Process. vol.1 (4), pp. 496-504, 1992.

[10] A. E. Savakis, and H. J. Trussell, "Blur identification by residual spectral matching", IEEE Trans. Image Process. vol. 2 (2), pp. 141-151, 1993. 
[11] D. G. Sheppard, H. Bobby, and M. Michael, "Iterative multiframe super-resolution algorithms for atmosphericturbulence-degraded imagery", J. Opt. Soc. Amer. A vol. 15 (4), 1998, pp. 978-992.

[12] Li, D., and Merserau, R. M. 2005. Blur identification based on kurtosis minimization. In Proceedings of the International Conference on Image Processing (ICIP), vol. 1, Genoa, Italy, 2005, pp. 905-908.

[13] D. P. Tuan, “An image restoration by fusion", Pattern Recognition Vol. 34, 2001. pp. 2403-2411.

[14] Rooms, F., Ronsse, M., Pizurica, A., and Philips, W. 2002. PSF Estimation with Applications in Autofocus and Image Restoration. In Proceedings of 3th IEEE Benelux Signal Processing Symposium (SPS-2002), Leuven, Belgium, March 21-22, pp. 13-16.

[15] B. S. Jeona, G. Choa, Y. Huhb, S. Jinb, and J. Park, "Determination of point spread function for a flat-panel $\mathrm{X}$ ray imager and its application in image restoration", Nuclear
Instruments and Methods in Physics Research A 563, 2006 , pp. 167-171.

[16] Shi, Y., and Eberhart, R. 1998. A modified particle swarm optimizer. In Proceedings of IEEE international conference on evolutionary computation, IEEE Press, Piscataway, NJ, 1998, pp. 69-73.

[17] Kennedy, J. 1997. The particle swarm: social adaptation of knowledge, In Proceedings of the IEEE international conference on evolutionary computation, IEEE Press, pp. 303-308.

[18] Kennedy, J., and Eberhart, R. 1998. Particle Swarm Optimization", In Proceedings of IEEE International Conference on Neural Networks, IEEE Press, Perth, Australia, vol. (4), pp. 1942-1948.

[19] M.R. Banham, and A.K. Katsaggelos, "Digital image restoration", IEEE Signal Process. Mag. vol. 14 (2), 1997, pp. 24-41. 